\title{
Augmented visualisation Designing experience for an interpretative cultural heritage
}

\author{
Bernadette Flynn \\ The Griffith Film Schoo1, Griffith University \\ b.flynn@griffith.edu.au
}

\begin{abstract}
The use of visualisation technologies is well established in digital cultural heritage. The earlier IT challenge of presenting material culture has largely been addressed with the production of high quality digital artefacts. A number of projects have drawn on the potential for augmented visualisation offered by mobile technologies, game engines and responsive environments. Alongside these advances is a recognition that a relevant digital cultural heritage needs to reflect contemporary interpretative practices rather than relying on outmoded systems of material science. While leading research in interpretative heritage has incorporated the hermeneutic aspects of previously lived cultures there have been less recognition of the importance of the users' role in the formation of cultural knowledge.
\end{abstract}

The paper proposes that we take a step back to investigate the processes of knowledge formation. It provokes a series of new research questions on visualizing cultural heritage knowledge in light of theoretical readings on perception and knowledge formation. It points to the need to devise alternative methods for the design and production of an interpretative digital cultural heritage. Such methods detail the generative potential of a complex process rather than the replication of a complex structure.

Keywords--- Digital heritage visualisation, phenomenological archaeology, interpretation, spatial praxis, somatic impulse.

\section{Experiential visualisation}

Interpretative digital cultural heritage is identified as an emerging theoretical and research practice area within digital heritage. Interpretative digital cultural heritage draws on contemporary practices in archaeology that foreground multi-vocal and culturally embedded interpretations of the past. Interpretative archaeology tries to bring the past to the human scale rather than considering it in an abstracted, detached way. Rather than relying on material science to understand the past interpretative archaeology employs the conceptual, the way people make sense of the world as social practice [1].

Within digital cultural heritage there has been an increasing drive to bring together these interpretative or experience-based accounts of the past with compelling user experiences. A key debate in the field has been around how the human dimensions of past experiences can be visualized in digital environments. UNESCO's cultural heritage charter recognises that a responsibility of heritage management is to reflect and represent not only the material culture of the past, but also intangible heritage as expressed through cultural activity and social practices pertaining to myths, oral traditions and ceremony.

In general traditional models for heritage visualisation has ill prepared designers to take up the challenge of representing experience-based accounts of the past. The role of visualisation in conveying the contextual experiences of the lived past has at times been taken as a give or considered unworthy of fuller exploration. The representation of knowledge associated with the duplication or reconstruction of cultural objects has frequently limited user interaction to access, retrieval and display of digital artefacts. Although the role of HCI design has gained prominence, the simulation of material evidence as the basis of knowledge has remained the priority in much digital cultural heritage practice.

\section{Social and cultural significance of digital cultural heritage}

The paper proposes the need for designing projects that move beyond the giveness of heritage as a digital representation of pre-existing or reconstructed material evidence. As such it proposes a rethinking of an interpretative agenda for digital cultural heritage. Interpretative archaeology has moved away from material science to propose that the process of interpretation is neither simple nor objective, but is constituted from multiple perspectives. For the interpretative archaeologist an understanding of the past is experientially derived through a process of embodied communication rather than presented. Central to this 
formulation is that material culture is produced from the dialogue between the past and the present and thus contextually derived and embedded in contemporary relationships.

Emerging responses to this interpretative turn in archaeology is evident across a number of digital heritage projects over the last decade. Researchers have moved beyond a simple equation between representation and meaning to inquire into the complex relationship between types of visualisation and cultural learning. Many have argued that knowledge of the past demands more than the verisimilitude of the visual display by paying attention to the affectivity of user participation. As Gillings notes visualisation methods by themselves are not enough to provide the user with an embedded experience of the past [2]. Efforts have been made to represent more experience based accounts of the past through such strategies as corpothetics [3]; peripatetic videowalks [4] and the representation of lived knowledge systems associated with the landscape and movement through the landscape [5].

While these emerging strategies have recognized the value of dynamic user participation for cultural learning, they have been perhaps less able to identify the users' role in knowledge formation. Forte, for instance, outlines that visual immersion and interaction are both important aspects in the integration of cultural values, while acknowledging that the precise nature of the relationship between representation and user experience remains uncertain [6]. The paper extends on this research by inquiring - what is the relationship between knowledge formation and material culture? How do these knowledge processes function for an interpretative digital cultural heritage?

\section{Phenomenological knowledge processes}

One avenue for reinstating an interpretative digital heritage is to draw on an established body of archaeological enquiry, which specifically theorizes how knowledge processes function. Phenomenological research in archaeology has been debated since the early 1980 s and has reached a stage of maturity, which references the complexity of the interpretative process and attends to the embodied aspects of representing the past.

A number of arguments for attending to the embodied aspects of user engagement have been previously established by the author [7-9]. Rather than reiterating these arguments, the paper explores how knowledge formation functions within an interpretative archaeology. It describes a methodology for using models of perception drawn from phenomenological archaeology [10-12]. It frames these through interpretations of the landscape and the application of a somatic praxis distilled from movement based systems of inquiry. Related to this, but outside the scope of this paper is an investigation into the phenomenology of responsive systems to identify how technologies might be used to enable the generative potential of somatic immersion.

\subsection{A phenomenological model of perception}

'A phenomenological perspective provides an ontological ground for the study of things, places and landscapes, a means of approach and a way of thinking through the body in its' participatory relation with the world' [12].

In phenomenological archaeology embodiment is taken as the starting point for analysing human participation in a cultural world [11-14]. Bodily activity within material space functions as an interpretative device for understanding the past and also by extension an expressive device [15]. In this framework, interpretation is a practice grounded in the body and archaeological knowledge arises from this interpretation. Typically, phenomenological frameworks have been applied to interpretations of British and European prehistory focusing on the experience of ritual or monumental landscape through sensory bodily devices [15].

This archaeological framework is of relevance for digital cultural heritage. Rather than behaviorist models of learning, which presuppose mechanistic transfer of knowledge, phenomenological models of perception assume that knowledge emerges from embodied experience. This embodied experience is defined by perceptual experience, modes of presence and engagement in a world [10]. For digital cultural heritage, such issues as encounter, performance and spatial navigation then become critical in conceptualising and designing the parameters of digital space and user engagement.

\subsubsection{Heightened attention}

The paper now turns to the important work by Csordas on perception as an embodied aspect of knowledge formation [10]. Csordas, brings together the writings of Merleau-Ponty on the phenomenology of perception and Bourdieu on the body as the locus of social practice to describe a somatic mode of attention. For Csordas, a somatic mode of attention is a 'culturally elaborated ways of attending to and with one's body in surroundings that includes the embodied presence of others' $[10$, p.138].

Csordas positions attention as fundamentally somatic (relating to or affecting the body) in that it involves a turning towards - a way of paying attention with ones body to the phenomenal world. In relation to this, Csordas argues that particular cultural modes of attention - processes in which we attend to and objectify our bodies - can be learnt and that our selves are constituted in and through socially meaningful embodied 
experiences. His somatic modes of attention are both conscious processes of knowledge generation and habituated through cultural patterning. Drawing on his observations on the processes of meditation or dance performance Csordas identifies that it is not just what bodies do but how they feel that generates a somatic mode of attention.

The framework advanced by Csordas's research suggests how attending to ones body functions as a process of knowledge production. Put simply 'attention can tell us something about the world and other things that surround us' [10, p. 139]. Knowledge of a past culture emerges from bodily engagement with the world in such a way that the material form of, for instance, rocks and figurines, along with the intangible aspects of a cultural heritage are enmeshed with human corporeality. The richness of a cultural heritage experience emerges from being able to enter into a framework of understanding associated with these experiences. 'To understand is ultimately always to construct, to constitute, to bring about here and now the synthesis of the object' $[16$, p.80].

\subsection{Spatial practice}

Central to a phenomenological archaeology is an emphasis on movement. The world is seen as composed of relationships, grounded in the sensuous embodied relation between persons and things. Phenomenological archaeology articulates the nature of encounter between human and artefact suggesting that the process of knowledge production emerges from performance or enactment. Such an understanding has particular relevance for extant buildings and architecture where the spatial affect of bodies in the heritage setting can be articulated through somatic engagement.

In relation to prehistory, Tilley outlines how the effects of landscape and architecture on the movements of people are generated through the human body and the sensory perceptions of persons [12]. Fundamental to any meaning making function is spatial motility and an attitude or posture towards objects. Modes of bodily engagement, such as, navigation and tactile apprehension of rocks and artefacts are processes for producing spatialized understandings. These spatialized understandings involve reciprocity between the body and the world - an active relationship where places affect bodies and bodies in turn affect perception.

For Tilley, the space of the world rather than being reducible to an objective and geometric grid when considered somatically is the space of sensory experience and bodily movement [11]. Any experience of space is grounded in the body itself, its capabilities and potentials for movement. Through time-space routines of movement and praxis or bodily practice a person knows where she or he is in relation to familiar places and objects and how to 'go on' in the world. Lived body- space incorporates not only habituated movement as 'habitual and unselfconscious action' but also modes of walking, turning, reaching, crouching, the performance of particular acts and body movements [11, p.16].

An emphasis for Tilley is on the body in the form of somatic space, which takes as its starting point the upright human body looking out onto the world. Tilley adopts movement categories from gestalt of front/back, vertical/horizontal, left/right or up/down and here/there polarities arguing that these movements are foundational to all human experience. These bodily dyads form the axes of spatial orientation which impose 'a schema on space through which it may be experienced and understood' [11, p.16]. Applying this to heritage, the visitor engages with a site from an embodied spatial location. The perceiving subject approaches and conceives of the world as a lived, dynamic field of potentiality. This approach operates as intentionality towards an object, which depends on who is experiencing it and how it is created, reproduced and transformed in relation to previous spatial interpretations.

In this action of intentionality motion, vision and comprehension are inseparable. What is being proposed is the unity of the whole body in movement where reasoning and conceptualisation, as much as vision, sight and smell are embodied processes. Such a phenomenology of perception and attention establishes the affect of the human body on the production of knowledge. In the encounter between subject and pregiven world things and landscapes alter our consciousness and constitute us beyond ourselves. Thus our own embodiment provides a way of opening up a dialogue with a past cultural horizon, which produces knowledge of material culture.

\section{Landscape and knowledge formation}

Following recent trends in archaeology and critical studies digital heritage scholars have taken up a phenomenological concept of landscape. As a way of rethinking spatial bodily inhabitation the landscape has been used to describe embodied sets of relationships linked to human feeling, movement and dwelling [11, 17]. As Tim Ingold points out, the perception of the environment proceeds along patterns of activity and observation leading to the continuous formation of the environment. Through the engagement of the mobile actor 'we know as we go from place to place' [17,p. 230]. Rather than the location of geographical objects within an imaginary birds-eye view of the landscape or single universal system of spatial coordinates landscapes are created and experienced through the manner in which they are explored and sensed, approached and left [12].

From this framework, walking is a process of knowledge transfer where consciousness is shaped by the spaces it passes through, and where consciousness in turn shapes 
the spaces it passes through. These landscapes then are not merely sites for viewing but are spaces of bodily motility and exploration 'invested with mythological understandings and ritual knowledges intimately linked with bodily routines and practices' [11, p.22]. In this way navigating a terrain, is a dynamic constellation between bodies, imagination and space.

This idea echoes De Certeau's notion of walking as an act of practice in the world where movement through space generates different types of understandings [18]. Tactics and strategies deployed by the walker form modes of spatial expression. Walking is thus an act of speaking the language of the terrain and through the improvised movements of the walker spatial elements are transformed or abandoned. Through the activity of walking, space (an unbounded notion) becomes a practiced place (made familiar through memory and familiar modes of encounter).

Grima and Bradley introduce a cosmological function to landscape encounter by suggesting that spatial praxis in relation to the heritage of monuments and landscape sites allows for a potentialization of the past $[19,20]$. The multiple changing functions of landscape as performed over time by the mobile body are designated as a potent medium of knowledge. As Grima notes, this may lead to, for instance, an understanding of tasks and activities that evoke symbolic and ritual meaning [21]

\section{An interpretative methodology for cultural heritage}

The phenomenological frameworks outlined so far in the paper draws attention to spatial practice as a necessary part of the archaeological process. What is referring to is an encounter between places, landscape and the body and the affects of that encounter. For Tilley and other archaeologists walking the landscape is then proposed as an integral aspect of the archaeological interpretative process. Tilley suggests archaeologists should themselves walk through the landscapes, which still contain traces of prehistoric monuments. Tilley's embodied exploration across Megalithic remains in the Welsh Black Mountains and the Dorset Cursus forms an elaborate reading of the heritage environment. His reading of the heritage environment moves past a visualist methodology to argue that past phenomenologies of the body can be understood through the body as interpretative apparatus. As outlined by Thomas our own embodied experience becomes analogous for those of past people [13]. The somatics of movement and gesture reveals 'the invisible in the visible, the intangible in the tangible' [12, p. 30].

As Shanks and Tilley suggest bodily experiences involve a stretching out of the past in the present in the sense that past experiences and structures are carried into the future
[22]. Bodily experiences of the landscape then necessarily involve translating from present day condition to the past. Feminist academics and cultural theoreticians have pointed out the dangers of universalising bodily experience. Acknowledging the contingency of cultural experiences, Thomas opines that our own selves do however provide a point from which we can engage with the past. He proposes that the most we can do is to experience and interpret prehistoric artefacts and landscape through our own embodiment and our own subjectivities and cultural positioning knowing that we create contemporary experiences [13].

The implications of Tilley's interpretative methodology is that bodily spatial praxis can operate as a mode of interpretation. Paying attention with the body and to the body creates a relationship with heritage monuments and artefacts that articulates cultural heritage at the level of the sensing, feeling, body. Cultural knowledge is made perceptual through the materiality and sensory corporeality of the body as an aspect of the interpretational process. Rather than thinking that this provides a fixed truth about the past, our embodiment provides a way of opening up a dialogue with a past cultural horizon [13].

The interpretative methodology outlined above suggests that cultural heritage might attend to the ways in which varying forms of bodily practice can provide an embodied approach to accessing the past. Following Tilley's method of walking the landscape suggests it is proposed that digital cultural heritage researchers actively engage with heritage space - that knowledge emerges from body-centered real world experience. If we take it that cultural knowledge is perceptual through body performance that it behooves us to engage directly with bodily process of learning.

Such a process demands a digital media process that operates as embodied interpretative practice through all stages of the knowledge chain. A preliminary proposal for including embodied interpretative practice is outlined below:

1. The lived body functions as a methodological starting point for an understanding of encounter between monument and user.

2. The capturing of somatic gesture as the material evidence of a somatic knowledge system.

3. The translation of body-centered real world experiences into modes of interaction

4. The identification of technologies and representational treatments that articulate the generative potential of emergent embodied processes.

\section{Body performance}

This section of the paper seeks to attend to the proposal for an embodied interpretative practice. It 
briefly advances a process for capturing somatic impulses made evident by performance. Documenting movement provides a methodological starting point and a foundation for articulating movement in the heritage site and its translation to the affective responsive engagement of users. It outlines how Tilley's notion walking the landscape as formative archaeological process might be extended to incorporate movement based expertise. A prehistoric structure is utilized as an example of somatic knowledge formation. The aim is to record a carnal or sensuous engagement with space that emerges from experiential movement and bodily gestures.

The first stage is to translate the phenomenological foundations of interpretative archaeology into a design methodology. An investigation of somatic praxis such as mind body centering techniques and ideokinesis offers some possibilities [23, 24]. Bonnie Bainbridge Cohen's somatic methodology uses the intentionality of the sensing, feeling and acting body. This provides an entry point into an understanding of how prehistoric architecture might be explored [23].

The somatic method outlines the encounter between the human body and prehistoric architectural space. This approach is characterized by an awareness of movement as an essential component in an encounter with the spatial matrix of the site. The movement practitioners 'map' the environment through developing a sensitivity and receptivity to the morphology and the sensuous qualities of the site. Movement is initiated by an impulse towards an element in the landscape, which might be a portal doorway, the texture of globigerina limestone, an artefact of the floor, the play of light and shade. The environment explored through bodily navigation is experienced as a place for contemplation, fluid or disjointed movement, dance or other performative acts rather than something merely gazed upon.

The process for identifying somatic responses to space focuses on capturing and analyzing the specifics of body movement in the heritage environment. High quality video recording of the movements is proposed to document the movement of the performers body in the heritage space. This details specific inflections or gesture patterns in relation to architectural morphology. Analysis of these recordings provides a database of key movements in the heritage environment.

For digital design the challenge is to translate the movements of people through the landscape and its consequent effects on spatiality into digital interaction scenarios. The intention is to create an environment where the mobile interaction of the body in encountering the heritage environment forms a framework for user experience. Taking account of and analysing 'performers' embodied experience provides a template for the design of interaction scenarios for the users movement in the digital environment. The emerging question is how the spatiotemporal characteristics of an archaeological site might be displayed in relation to the living moving bodies of visitors in the virtual environment. One response to this is to explore the phenomenological investigations of new media artists working with interaction visualisation technologies. The work by artists such as Char Davies and David Rokeby suggests further avenues for productive exploration [2527]. Further discussion of digital design using immersive technologies is outside the parameters of this paper and will be addressed in later publications.

\section{Conclusion}

The paper has set out to investigate the processes of knowledge formation in an interpretative digital cultural heritage. It has argued that in order to redefine a cultural agenda for heritage visualisation we need to establish how aspects of cultural knowledge are embodied. That is - to identify how the body, as both interpretational constraint and enabling condition informs present day understandings of the past.

The paper has pointed out the limitations of assuming a straightforward transfer of cultural heritage knowledge between the user and the digital representation. It argues that cultural knowledge is a complex process irreducible to the parameters of a visualisation model. It suggests that the past is culturally constructed as well as lived and experienced. The paper proposes a methodology for capturing the varying forms of bodily practice and somatic encounter. It outlines how the user is an active participation in the processes of knowledge formation and how interpretative practice from a phenomenological archaeology might be leveraged for the design of a digital cultural heritage environment. Overall, the paper throws up a number of questions rather than providing answers. Many of these require further theoretical development and attention through the trialing of methods of practice and prototype development.

\section{References}

[1] I. Hodder, Theory and Practice in Archaeology. London and New York: Routledge, 1992.

[2] M. Gillings, "The Real, the Virtually Real, and the Hyperreal: The Role of VR in Archaeology," in Envisioning the Past: Archaeology and the Image, S. Smiles and S. Moser, Eds. Oxford: Blackwell Publishing, 2005, pp. 223-239.

[3] S. Kenderdine, "Somatic solidarity, Magical Realism and Animating Popular Gods: Place-Hampi ' where intensities are felt'," IEEE Multimedia, 2007.

[4] R. Tringham and M. Ashley, "Senses of Places: Remediations from Text to Digital Performance," 2005.

[5] B. Leavy, J. Hills, C. Barker, and S. Gard, "Digital Songlines: Digitising the Arts, Culture and Heritage Landscapes of Aboriginal People," in New Heritage Forum, Hong Kong, 2006.

[6] M. Forte, "Ecological Cybernetics, Virtual Reality, and Virtual Heritage," in Theorizing Digital Cultural Heritage: A Critical Discourse, F. Cameron and S. Kenderdine, Eds. Massachusetts: The MIT Press, 2007, pp. 389-407. 
[7] B. Flynn, "Prehistoric Archaeology in a Dynamic Spatial Visualization Environment," 2006.

[8] B. Flynn, "Somatic Knowledge and Simulated Spaces," in 11th international Congress: Cultural Heritage and New Technologies, Vienna, 2006.

[9] B. Flynn, "Digital Knowledge as Archaeological Spatial Praxis," in 13th International Virtual Systems and Mutlimedia Conference, Brisbane, 2007.

[10] T. J. Csordas, "Somatic Modes of Attention," Cultural Anthropology, vol. 8, pp. 135-156, 1993.

[11] C. Tilley, A Phenomenology of Landscape: Places, Paths and Monuments. Oxford: Berg, 1994.

[12] C. Tilley, The Materiality of Stone: Explorations in Landscape Phenomenology. Oxford: Berg, 2004.

[13] J. Thomas, "The Great Dark Book: Archaeology, Experience, and Interpretation," in A Companion to Archaeology, T. Earle and C. S. Peebles, Eds.: Blackwell Publishing, 2004.

[14] R. Tringham, "Engendered Places in Prehistory," in Interpretive Archaeology, J. Thomas, Ed. London and New York: Leicester University Press, 2000.

[15] Y. Hamilakis, M. Pluciennik, and S. Tarlow, "Introduction: Thinking through the Body," in Thinking through the Body: Archaeologies of Corporeality, Y. Hamilakis, M. Pluciennik, and S. Tarlow, Eds. New York: Kluwer Academic/Plenum Publishers, 2002, pp. 1-21.

[16] M. Reuter, "Merleu-Ponty's Notion of Pre-Reflective Intentionality," Synthese, vol. 118, pp. 69-88, 1999.

[17] T. Ingold, The Perception of The Environment: Essays in Livelihood, Dwelling and Skill. London: Routledge, 2000.

[18] M. DeCerteau, The practice of everyday life. Berkley: University of California Press, 1984.

[19] R. Grima, "Monuments in Search of a Landscape: The Landscape Context of Monumentality in Late Neolithic Malta," in Institute of Archaeology London: University College London, 2005.

[20] R. Bradley, The Past in Prehistoric Societies. London: Routledge, 2002.

[21] R. Grima, "An Iconography of Insularity: A Cosmological Interpretation of some Images and Spaces in the Late Neolithic Temples of Malta," Papers from the Institute of Archaeology, pp. 48-65, 2001.

[22] M. Shanks and C. Tilley, Re-Constructing Archaeology: Theory and Practice. London and New York: Routledge, 1992.

[23] B. B. Cohen, Sensing, Feeling and action The Experiential anatomy of Body-Mind Centering. Northhampton, MA: Contact Editions, 1993.

[24] M. Todd, The Thinking Body. New York: Dance Horizons, 1972.

[25] J. a. W. Shaw, Peter, Future Cinema: the Cinematic Imaginary after Film. Cambridge, Massachusetts: ZKM and The MIT Press, 2003.

[26] M. Hansen, New Philosophy for New Media. Cambridge, M.A.: The MIT press, 2004.

[27] D. Rokeby, "The Construction of Experience : Interface as Content," in Digital Illusion: Entertaining the Future with High Technology, J. Clark Dodsworth, Ed.: Addison-Wesley Publishing Company, 1998 